\title{
Validity of bioluminescence measurements for noninvasive in vivo imaging of tumor load in small animals
}

\author{
Clara P.W. Klerk1, Renée M. Overmeer1, Tatjana M.H. Niers¹, Henri H. Versteeg1, \\ Dick J. Richel1, Tessa Buckle², Cornelis J.F. Van Noorden1, and Olaf van Tellingen²
}

BioTechniques 43:S7-S13, S30 (July 2007)

doi 10.2144/000112515

\begin{abstract}
A relatively new strategy to longitudinally monitor tumor load in intact animals and the effects of therapy is noninvasive bioluminescence imaging (BLI). The validity of BLI for quantitative assessment of tumor load in small animals is critically evaluated in the present review. Cancer cells are grafted in mice or rats after transfection with a luciferase gene-usually that of a firefly. To determine tumor load, animals receive the substrate agent luciferin intraperitoneally, which luciferase converts into oxyluciferin in an ATP-dependent manner. Light emitted by oxyluciferin in viable cancer cells is captured noninvasively with a highly sensitive charge-coupled device (CCD) camera. Validation studies indicate that BLI is useful to determine tumor load in the course of time, with each animal serving as its own reference. BLI is rapid, easy to perform, and sensitive. It can detect tumor load shortly after inoculation, even when relatively few cancer cells (2500-10,000) are used. BLI is less suited for the determination of absolute tumor mass in an animal because of quenching of bioluminescence by tissue components and the exact location of tumors because its spatial resolution is limited. Nevertheless, BLI is a powerful tool for high-throughput longitudinal monitoring of tumor load in small animals and allows the implementation of more advanced orthotopic tumor models in therapy intervention studies with almost the same simplicity as when measuring traditional ectopic subcutaneous models in combination with calipers.
\end{abstract}

\section{INTRODUCTION}

Animal models remain important experimental tools to investigate mechanisms of tumor development and effects of therapy. Since cancer progression consists of a wide variety of events, such as primary tumor growth, angiogenesis, invasion into surrounding tissues, extravasation of cancer cells, and growth of metastases $(1,2)$, it is likely that (potential) therapies may be effective at certain stages of cancer progression only. Thus far, assessment of the effects of therapeutic intervention has usually been made using subcutaneously implanted tumors, which, however, do not recapitulate many of the essential features of tumor growth in patients (3). Whereas orthotopically implanted tumors mimic the clinical situation more closely and may be preferred $(4,5)$, assessment of such tumor burden initially required the sacrifice of the animals at a certain time point after cancer cell inoculation and evaluation of tumor load at time of death. The course of tumor development in time was then assessed by comparing groups of animals that were sacrificed at different time points. Because of substantial inter-individual variation, large numbers of animals and laborious efforts were required, rendering the use of orthotopic models highly impractible (6). Noninvasive determination of tumor load over time in individual animals can reduce the numbers of animals in experiments considerably and provide information on the various stages of tumor development (7-9). Alternatively, tumor load in animals is determined by surgically opening the animals to inspect the organ affected by cancer (10), but this type of procedure causes considerable discomfort to the animal and may also affect cancer progression by operation- related effects such as activation of the coagulation cascade (11).

Tumors that are not visible or palpable noninvasively can also be visualized by special preparation of tissues. Skin-fold chambers and subcutaneous windows inserted with semitransparent material can be used to visualize tumor development at a cellular or even subcellular level for longer periods of time $(12,13)$. These techniques can also be combined with externalization of organs of interest (14). However, the maintenance of windows for longer periods of time is rather difficult (14). Furthermore, investigations are limited to areas exposed by the chamber or window. The use of skin flap models can extend the areas of investigation considerably (15). Again, these approaches have the disadvantage that heterotopic tumors are used and operation-related effects may affect tumor development.

${ }^{1}$ Academic Medical Center, University of Amsterdam, Amsterdam and 2 The Netherlands Cancer Institute/Antoni van Leeuwenhoek Hospital, Amsterdam, The Netherlands 


\section{Imaging Frontiers}
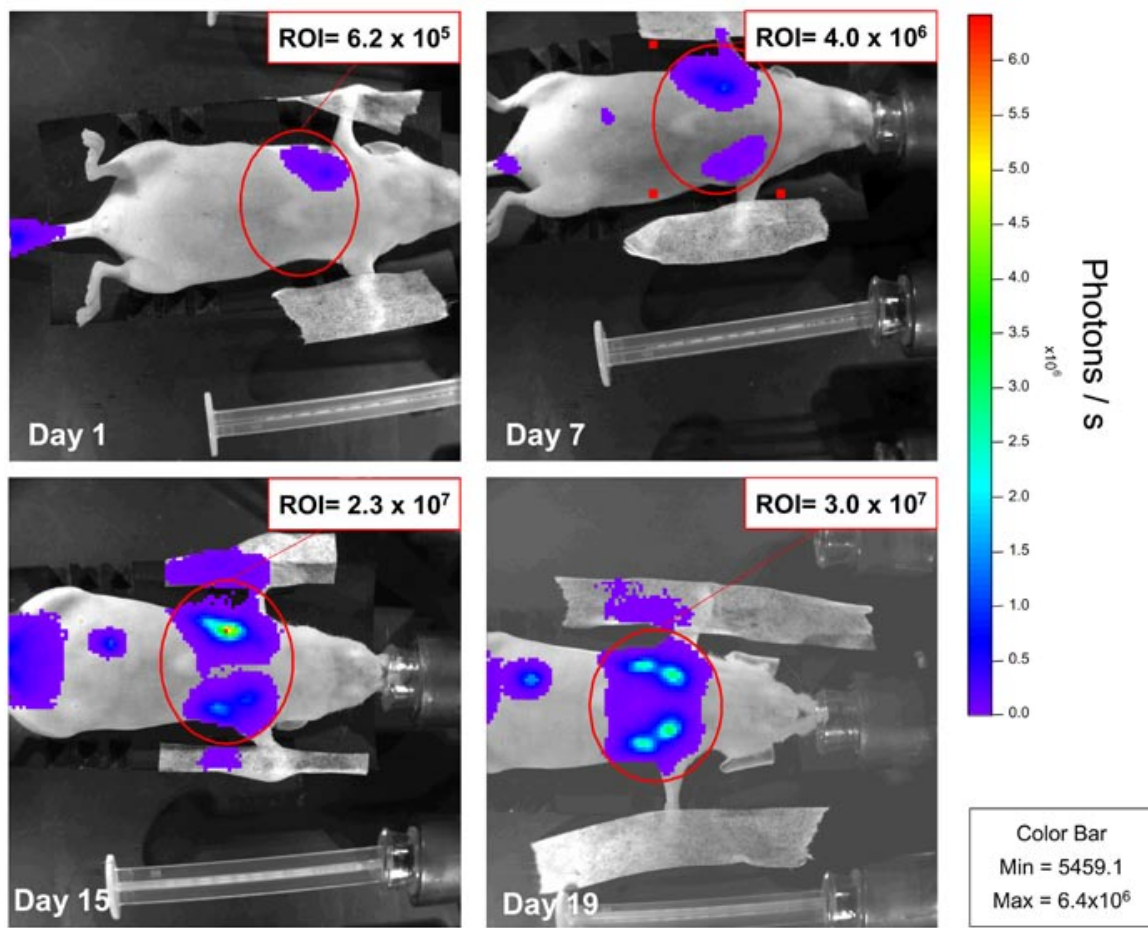

Figure 1. Noninvasive bioluminescence imaging (BLI) of an individual mouse in time after intravenous administration of luciferase-expressing melanoma cells. This mouse received $1 \times 10^{6}$ K1735Br2lucA3 melanoma cells expressing luciferase at day 0 . Bioluminescence generated by luciferase activity was visualized using a supercooled charge-coupled device (CCD) camera. For this purpose, the animal was given $150 \mathrm{mg} / \mathrm{kg}$ luciferin intraperitoneally and 10 to $15 \mathrm{~min}$ later was anesthetized using isoflurane and placed in a thermostated dark box under the camera. Immediately after the acquisition of the white light image, a dark image was taken for $60 \mathrm{~s}$ or less (depending on the signal intensity) using maximal lens aperture. Images were taken at days 1, 7, 15, and 19 after inoculation of the melanoma cells. Both the white light and the dark images were merged by the software to allow easy orientation of the tumor mass. Each dot represents a cluster of CCD pixels, and the intensity of each dot is depicted on a false color scale. The drawn ovals demarcate the region of interest (ROI) that was selected for the quantification of the bioluminescence signal (in photons/s) in the lungs. The mouse also shows bioluminescence in the tail and the abdomen, indicating the presence of cells at the site where cancer cells were inoculated and cancer cells spread into the abdomimal region. Also note the reflection of light on the tape and the paws that become visible especially with very bright images.

\section{NONINVASIVE IMAGING}

Several noninvasive approaches to imaging tumor development in small animals have become available, such as magnetic resonance imaging (MRI), computerized tomography (CT), positron emission tomography (PET), single photon emission computed tomography (SPECT), and ultrasonography. Each approach has its own advantages and disadvantages. MRI, CT, and ultrasonography produce anatomical images of structures in the body, whereas PET and SPECT image physiological processes and thus produce functional images. Their use for the in vivo study of cancer progression in small animals has been reviewed recently $(7,8,16)$. Alternatively, the use of two relatively new noninvasive approaches, fluorescence imaging (FLI) and bioluminescence imaging (BLI), is expanding rapidly.

\section{NONINVASIVE FLI}

For FLI, cancer cells are transfected with the gene for green fluorescent protein (GFP) or related fluorescent proteins that allow visualization of these cells in living animals using a sensitive charge-coupled device (CCD) camera (17-19). Large tumors can even be observed noninvasively with the naked eye $(20,21)$. Other reporter genes have been applied as well that make cells fluorescent when a specific promoter is activated (22) or encode for an enzyme, such as $\beta$-galactosidase, that produces a fluorescent product (19). The use of skin flaps in combination with highpower microscopes even allows investigation of these tumors at a single-cell level (15). Additionally, nonfluorescent cells or tissue compartments can be studied against a fluorescent background of the cancer cells, as they contrast with this background. In this way, host (immune) cells and angiogenesis have been studied in tumors $(15,17,23)$. A disadvantage is the fact that cancer cells have to be transfected with a reporter gene and that may alter their malignant behavior. Moreover, visualization of fluorescent cancer cells in the deeper parts of animals that cannot easily be exposed is often hard or even impossible to accomplish because of interference by autofluorescence and quenching of excitation and emission light by tissues (7). Excitation light and emission light are often of wavelengths below $600 \mathrm{~nm}$, and at these wavelengths, tissue components quench strongly $(21,24,25)$. Light at wavelengths between 600 and 900 $\mathrm{nm}$ is more optimal for use in live animals, as tissues are more translucent $(8,19,26)$ and produce less autofluorescence $(8,16,21,24)$. However, the food in the gastrointestinal tract may contain plant products that are fluorescent at longer wavelengths and thus interfere with the sensitivity of FLI (24).

\section{NONINVASIVE BLI}

In general, BLI is based on ATPdependent conversion of luciferin by luciferase into the light-emitting product oxyluciferin. Cancer cells transfected with a luciferase gene of the firefly Photinus pyralis are inoculated, and subsequent bioluminescence measurements after administration of the substrate, luciferin, provide information on the amount of luciferase activity and thus cancer cells in the animal $(7,27-29)$. It has been shown that BLI can be used to track cells anywhere in the body following the intraperitoneal administration of a saturating dose of luciferin, including potential sanctuary sites like the brain $(30,31)$. ATP that is required for the enzyme reaction is endogenously present in (viable) cancer cells. The 
Table 1. Sensitivity and Validity of BLI Measurements of Tumor Load in Small Animals Using Luciferase-Espressing Cancer Cells

\begin{tabular}{|c|c|c|c|c|c|}
\hline Cancer Cell Type & Site of Tumor & Solid Mass or Diffuse Tumors & Sensitivity & Validity & Reference \\
\hline \multirow[t]{3}{*}{ HeLa } & s.c. & Solid mass & $1 \times 10^{4}$ & + & 27 \\
\hline & i.p. & Diffuse & $1 \times 10^{3}$ & + & 27 \\
\hline & i.v. & Diffuse & $1 \times 10^{6}$ & - & 27 \\
\hline \multirow[t]{2}{*}{ HeLa } & i.p. & Diffuse & $2.5 \times 10^{3}$ & +++ & 28,48 \\
\hline & i.m. & Solid mass & $1 \times 10^{3}$ & ++ & 28,48 \\
\hline Gliosarcoma & Orthotopic & Solid mass & & +++ & 43 \\
\hline Glioma & Orthotopic & Solid mass & $1 \times 10^{5}$ & +++ & 39 \\
\hline Glioma & Orthotopic & Solid mass & & + & 31 \\
\hline Glioma & Orthotopic & Solid mass & & ++ & 30 \\
\hline Pituitary & Spontaneous & Solid mass & & +++ & 37 \\
\hline Prostate cancer & i.p. & Diffuse & $1 \times 10^{3}$ & ++ & 33 \\
\hline \multirow[t]{2}{*}{ Prostate cancer } & s.c. & Solid mass & & +++ & 41 \\
\hline & Orthotopic & Solid mass & & +++ & 41 \\
\hline Lung cancer & i.v. & Diffuse & & ++ & 41 \\
\hline Prostate cancer & s.c. & Solid mass & $4 \times 10^{2}$ & & 24 \\
\hline HeLa & i.m. & Solid mass & $9 \times 10^{2}$ & & 24 \\
\hline \multirow{3}{*}{$\begin{array}{l}\text { Mammary } \\
\text { carcinoma }\end{array}$} & $\mathrm{bm}$ & Solid mass & $2 \times 10^{4}$ & & 35 \\
\hline & i.m. & Solid mass & $1 \times 10^{4}$ & & 35 \\
\hline & s.c. & Solid mass & $1 \times 10^{3}$ & & 35 \\
\hline Colon cancer & i.p. & Diffuse & & ++ & 9 \\
\hline Lymphoma & i.v. & Diffuse & $7 \times 10^{3}$ & +++ & 40 \\
\hline
\end{tabular}

bioluminescence signal increases during the first minutes, reaching a plateau after about 10 to $15 \mathrm{~min}$, and remains constant until approximately $40 \mathrm{~min}$ after luciferin injection (31). Consequently, a safe time frame to read the BLI signal is within 20-30 $\min (30,31)$. There are a number of alternatives for luciferase from other organisms to produce bioluminescence $(28,32)$, but BLI with firefly luciferase remains the most obvious option at present because of its spectral characteristics and the pricing of the substrate.

Noninvasive BLI is easy to perform and is not affected by movements in the body due to heartbeat and breathing. The downside of BLI is the low spatial resolution $(7,8)$ (compare Figures 1 and 2). However, spatial resolution can be obtained afterwards by macroscopical (Figure 2) or microscopical (Figure 3) inspection of relevant tissues guided by BLI images (Figure 1). Another disadvantage of BLI is that cancer cells need to be genetically modified in order to carry the luciferase gene, which makes a direct translation of data in animal studies to the clinic impossible. On the other hand, noninvasive BLI can be far more sensitive than any of the other noninvasive techniques. It is the only approach that can image cancer cell load from the moment that cancer cells are administered to the animals (Figures 1 and 4). Moreover, the formation of bioluminescence is ATP-dependent, and therefore only metabolically active cancer cells contribute to bioluminescence production, and as such, the bioluminescence signal is only derived from living cancer cells and not from necrotic areas in tumors $(7,28)$.

BLI is also more sensitive than FLI because it does not require an external excitation light source $(21,24,25,33)$. BLI is performed in absolute darkness, thus avoiding the interference with background light and autofluorescence, making the measurement of small lesions more reliable $(16,21,24)$.
Furthermore, only the emitted light produced in BLI has to travel through quenching tissue layers. Quenching of the signal by tissue components does occur, but at an acceptable level. Bioluminescence of oxyluciferin has a broad spectrum with a large component above $600 \mathrm{~nm}$ where hemoglobin and melanin (primary light quenchers in tissues) absorb relatively little light (28). Hair scatters light, and although nude mice may be preferred for BLI in this respect (21), useful results can also be obtained in hairy and pigmented mice. The technique has been successfully used to monitor cancer metastasis and the effects of antineoplastic therapy in animal models (31,33-39).

The relationship between the bioluminescence signal in intact animals and the viable cancer cell load has to be valid. In other words, the bioluminescence signal has to represent quantitatively the amount of cancer cells in the animals. Surprisingly, few studies have been devoted thus far to establish a 


\section{Imaging Frontiers}

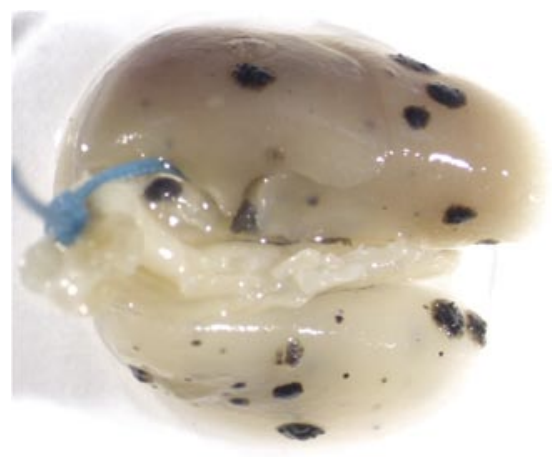

Figure 2. Image of an intact lung of a mouse at 19 days after melanoma cell inoculation in the tail vein. Tumors of B16F10 melanoma cells are visible as dark spots in the white lung tissue. When comparing the images in Figures 1 and 2 , the low spatial resolution of in vivo bioluminescence imaging (BLI) is obvious. After 19 days, animals were sacrificed. Immediately afterwards, the abdominal cavity was opened, and the inferior caval vein was cut in order to prevent blood flow into the chest cavity during operation. Then the chest cavity was opened, and the lungs were expanded by intratracheal administration of 0.7 $\mathrm{mL}$ solution of $6 \%(\mathrm{w} / \mathrm{v})$ polyvinyl alcohol (49) in phosphate-buffered saline (PBS) to keep the structure of the lungs intact. Subsequently, the trachea was closed by suture (blue thread), the lungs were removed, frozen in liquid nitrogen, and stored at $-80^{\circ} \mathrm{C}$ until ready for use.

direct quantitative relationship between the bioluminescence signal in live animals and their cancer cell load. The aim of the present review is the critical evaluation of validation studies of BLI in intact animals as a measure of viable cancer cell load. Because most studies used only superficially implanted solid tumors underneath the skin or skull, we have included data from a validation experiment in a melanoma metastasis model in which small tumors develop diffusely throughout the lungs (Figures 1 and 2).

\section{SENSITIVITY OF NONINVASIVE BLI}

Sensitivity of BLI is dependent on various factors, such as luciferase expression levels in the cancer cells, the site of implantation of the tumors (amount of tissue between the bioluminescent cancer cells and the camera), oxygenation and viability of the tumors, and sensitivity and settings of the camera (especially the clustering of pixels, as this determines the signal-
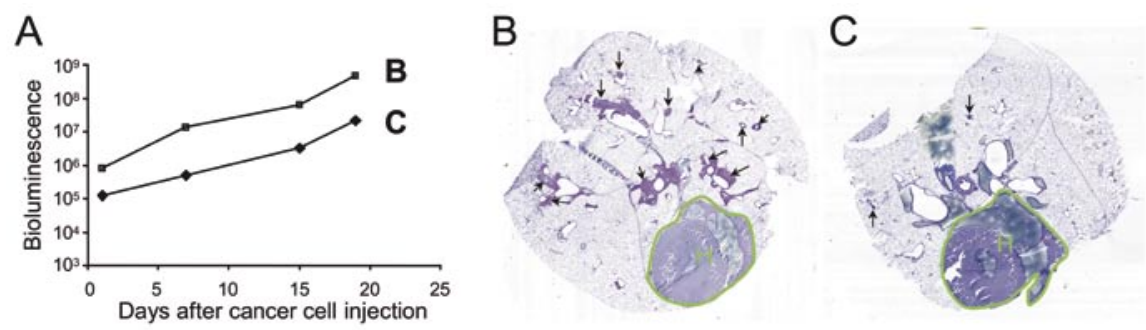

Figure 3. Bioluminescence imaging (BLI) data of two individual mice inoculated with luciferaseexpressing melanoma cells and lung sections of the mice showing tumors. (A) Bioluminescence was determined noninvasively at days $1,7,15$, and 19, and (B and C) images of sections of these lungs after Giemsa staining were taken at day 19 after killing the animals. One mouse shows high levels, and one mouse shows lower levels of bioluminescence at day 19. These noninvasively collected data correspond with the numbers of tumors (arrows) found in sections of the lungs.

to-noise ratio). For the most correct assessment of the sensitivity of BLI, the background levels of photon fluxes should be determined with the same system settings and used, for example, by taking five times the background as a minimum level of measurement. However, this procedure is often not performed.

Table 1 lists the studies that investigated sensitivity and validity of BLI to quantify tumor load noninvasively. It shows that sensitivity depends on the way cancer cells are inoculated: subcutaneously, intraperitoneally, or intravenously. When tumors are solid masses located close to the surface of the animal, sensitivity is highest. When cancer cells are diffusely present intraperitoneally, sensitivity is similar or somewhat less, whereas intravenous inoculation leads to the lowest sensitivity. When cells are inoculated orthotopically, intramuscularly, or intracranially to yield a local tumor mass, the sensitivity resembles that of solid masses that are inoculated subcutaneously.

The higher numbers of cancer cells that need to be inoculated systemically (intravenously) than more locally (subcutaneously or intraperitoneally) can be explained by the usually deeper tissue layers in which intravenously inoculated cancer cells grow, the more diffuse development of tumors after intravenous inoculation (Figures 2 and 3 ), and the lower grafting efficiency after intravenous inoculation in comparison with subcutaneous or intraperitoneal inoculation. Nevertheless, detection of 400 to1000 cancer cells inoculated subcutaneously or intraperitoneally and 1000-10,000 cancer cells inoculated intravenously appears to be feasible using BLI, which makes it the most sensitive noninvasive method yet available.

A number of studies compared the sensitivity of BLI with FLI and found that BLI was far more sensitive due to the much lower background $(21,24)$. A different setup, in which B cell lymphoma cells that expressed both luciferase and GFP were inoculated intravenously and were used to compare BLI data with numbers of GFP-labeled cancer cells in spleen and liver (major sites of cell proliferation) as detected after sacrifice by fluorescence-activated cell sorting (FACS) analysis, demonstrated that BLI was far more sensitive (40).

In conclusion, BLI is capable to detect low numbers of luciferaseexpressing cells under proper conditions, which is important for its implementation in therapy-intervention studies. However, BLI is not yet sensitive enough to track cells at the single-cell level.

\section{VALIDITY OF NONINVASIVE BLI}

The validity of noninvasive quantification of the BLI signal as a parameter for tumor load has been tested in various ways, and the outcome is shown in Table 1. BLI data were correlated with the amount of transfected tumor cells injected (intraperitoneally and intramuscularly) (33), with tumor volume measurements using calipers of subcutaneously inoculated cancer cells $(41,42)$, MRI volume measurements of intracranially growing tumors $(30,43)$, weight measurement of tumors 
$(37,42)$, and FACS analysis of cancer cells in affected tissues (40). In all cases in which tumors grew as relative uniform and/or well-confined lesions, good correlations were found between BLI data and tumor volume or tumor weight data. A good correlation was also reported between total lung weight and BLI data of metastatic lung tumors after intravenous inoculation of cancer cells in the tail vein (41). However, we were not able to confirm this when comparing noninvasive in vivo BLI data with ex vivo (in vitro) bioluminescence measurements of whole lung tissue lysates (Figure 5) or standard histological scoring (Figure 6) of diffuse lung tumors after intravenous inoculation of cancer cells in the tail vein. We plotted in vivo bioluminescence in lungs of mice at the day of sacrifice (day 19 after cancer cell injection) against the number of tumors present in the lungs on that day as scored by two microscopical approaches (Figure $6)$. The bioluminescence values at the day of sacrifice were found to correlate with both microscopical scoring methods [total number of tumors in the lungs versus only larger tumors in the lungs; coefficients, $0.47(P=0.04)$ and $0.80(P<0.001)$, respectively]. When the Pearson's correlation test for normally distributed data was applied to the logarithmic values, only the second microscopical scoring method was found to correlate in a statistically significant manner [coefficient, $0.76(P<0.001)]$. The finding that inclusion of very small lesions reduces the correlation is likely due to the fact that they contribute only minimally to the emission of light, whereas these smaller lesions contribute equally to the total count of lesions as the larger ones.

Most studies report moderate to good correlations between tumor mass and BLI signal. However, data of El Hilali et al. (33), who compared in vivo BLI data with ex vivo luminescence of tumor tissue lysates, suggest that the BLI intensity increases nonproportionally with tumor mass as determined by ex vivo bioluminescence in tissue lysates. The authors explained this phenomenon by proposing that larger tumors show increased optical density and thus increased quenching
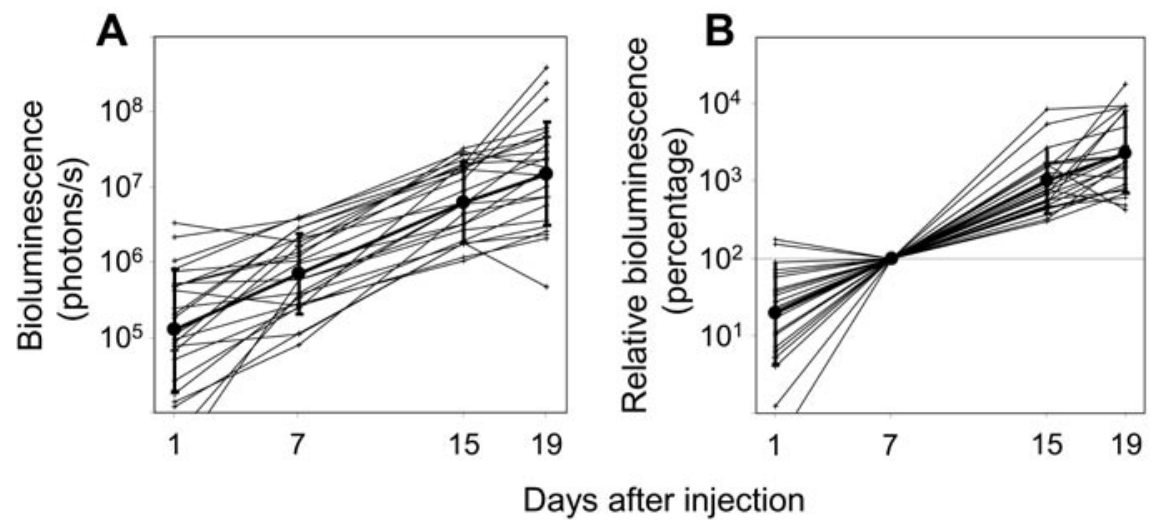

Figure 4. Bioluminescence imaging (BLI) data of 26 individual mice in time after intravenous administration of luciferase-expressing melanoma cells. (A) Mice were inoculated with $1 \times 10^{6}$ luciferase-expressing K1735Br2lucA3 melanoma cells, and bioluminescence was determined at days $1,7,15$, and 19 after inoculation. The bold line shows the mean bioluminescence \pm sD of this series of animals. (B) BLI data of the 26 mice shown in panel A normalized to the data on day 7 (set at 100\%).

of the signal and reduced availability of substrate to the tumor core. If this would prove to be a common phenomenon, it would imply that tumor load is underestimated when using BLI.

Importantly, however, BLI is mainly used for longitudinal assessment of tumor growth, for example in drug intervention studies, and not for single assessment of tumor burden. Therefore, it is possible to normalize the BLI signal relative to the BLI signal at the reference date (i.e., start of therapy). This normalization greatly reduces the inter-animal variation in BLI signal during the course of the study. When the position of the animal toward the camera is kept constant from measurement to measurement (Figure 1), inter-animal reproducibility of the relative BLI signal is acceptable (Figure 4B). On the basis of our own experience, a group size of eight test animals versus eight controls is sufficient, which is not different from the group size in experiments using subcutaneous tumor models.

\section{BLI IN THERAPY INTERVENTION STUDIES}

BLI is considered to be particularly useful for longitudinal follow-up of tumor growth during therapy intervention studies, especially since it allows the use of more sophisticated orthotopic tumor models instead of subcutaneous models. However, relatively few studies have used

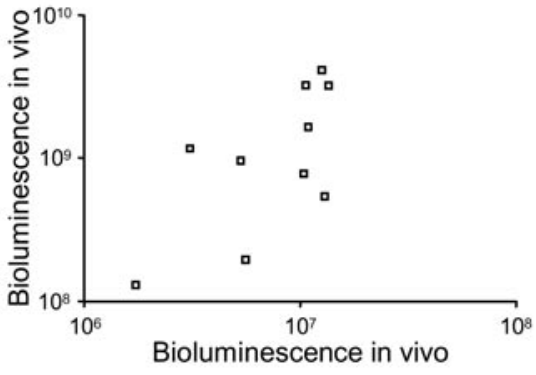

Figure 5. Correlation between in vivo and ex vivo bioluminescence data of lungs of mice containing melanoma metastases. Noninvasive bioluminescence data of 10 mice at day 19 after inoculation of $1 \times 10^{6}$ melanoma cells expressing luciferase and ex vivo bioluminescence data in homogenates of the same lungs prepared directly after the noninvasive measurement at day 19. Lung tissue was lyzed/homogenized in cell lysis buffer from the in vitro bioluminescence kit (Luciferase Assay System, Promega, Madison, WI, USA).

BLI for this purpose. Thus, it seems fair to state that the implementation of this methodology is still very much in its infancy.

When using BLI for intervention studies, the BLI signal just prior to initiation of the intervention (reference date) is determined. It can be used to stratify the animals into the control versus treatment groups with approximately similar mean BLI signals (i.e., tumor load). The BLI signal of subsequent images of each animal can be normalized relative to the reference BLI signal of the same animal. This normalization helps to reduce the inter-animal variation in the BLI signal during the course of the study. Obviously, the 


\section{Imaging Frontiers}
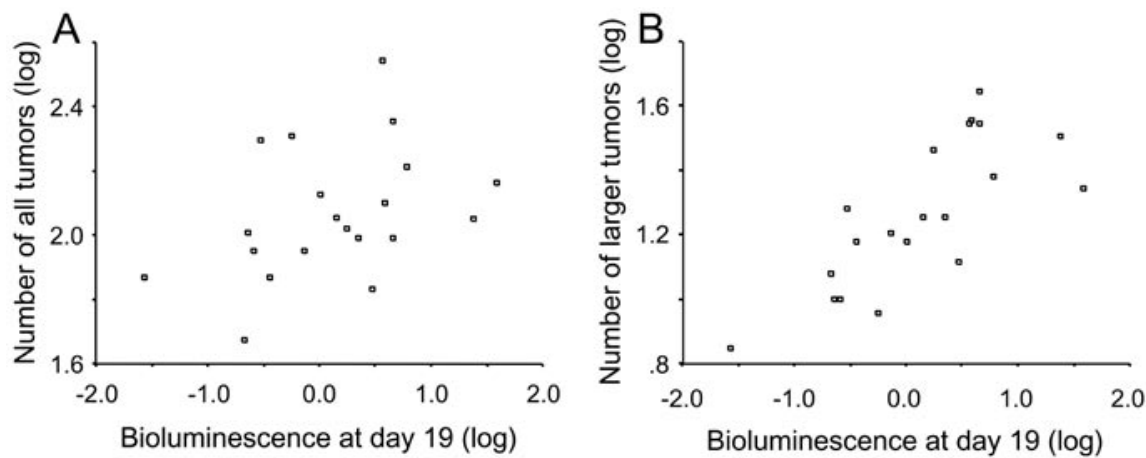

Figure 6. Correlation between the logarithmic bioluminescence data at day 19 and (A) logarithmic number of all tumors in the lungs irrespective of their size and (B) tumors that were at least 5 cells in diameter. Microscopic scoring was performed to determine tumor load. Sections $(6 \mu \mathrm{m}$ thick) of lungs of 20 mice shown in Figure 4 were cut using a motor-driven cryostat. To perform reproducible sampling that was indicative for the entire lungs, sectioning was performed starting at the base of the lungs, and sections $600 \mu \mathrm{m}$ apart were selected (50). Giemsa-stained sections were used for quantitative assessment of numbers of tumors. To allow comparison of adjacent sections (i.e., $600 \mu \mathrm{m}$ apart), overviews of the sections were prepared by scanning and printing digital images (50). Cancer cell clusters that were recognized microscopically were marked on these printouts. The marked printouts were compared, and tumors present in the same location in consecutive sections were counted once only. Two methods of scoring were applied. In the first method, every cluster of melanoma cells, however small, was considered as a tumor. In the second approach, only clusters or tumors of melanoma cells were counted that were at least 5 to 6 cells in diameter in the sections.

position of the animal toward the camera needs to be kept constant from measurement to measurement (as in Figure 1).

Veenendaal et al. (44) have successfully used this approach in a liver metastasis model using eight animals per study arm. However, it is important to realize that a liver metastasis model was used in which the tumor was implanted as a fragment onto the liver, resulting in a single focal lesion, rather than in disseminated spread of tumors throughout the entire organ as occurs after intra-splenic or portal vein injection. This focal growth may result in a more uniform relationship between BLI signal and tumor load. Similar results have been reported in other models that resulted in solitary lesions, such as brain tumors following stereotactic intracranial injection $(30,31)$ or bone lesions following injections of cancer cells in the femur or tibia $(45,46)$. Typically, approximately seven to ten mice per group were sufficient to detect relevant differences between test and control groups. Thus, BLI appears to fulfill its promise to enable the use of orthotopic models without the need to use substantially more animals than are used in subcutaneous models.

Similarly, BLI has been used for monitoring therapy efficacy against bone metastases originating from left ventricle cardiac injection, but here the lesions also developed as clear foci (45). However, the use of BLI for follow-up of interventions in disseminated disease such as lung or liver metastases may require more animals per group because of substantial interanimal variation in BLI signal. Taking into account our own results in the lung metastasis model (setting day 7 as the reference day for normalization), the SD of the BLI signal on subsequent days requires a group size of approximately thirteen animals to find a 5fold reduction in tumor growth with a power of 0.8 . In contrast, Dikmen et al. (47) using a similar lung metastasis model have used only three to four mice per group, but this was possible only because of a more than 100-fold difference between the treatment and control groups. Likewise, Saur et al. (36) monitoring liver and lung metastases used much larger groups but used BLI only to score absence and presence of metastases in these organs. Thus, in the case of solitary lesions, BLI appears to fulfill its promise to enable the use of orthotopic models without substantially increasing the number of animals relative to subcutaneous models. More animals are needed per group for follow-up of metastatic tumor growth. However, these numbers are still considerably smaller than those needed when the tumor burden is determined after sacrifice of the animals.

\section{CONCLUSIONS}

In conclusion, BLI is a powerful noninvasive tool for longitudinal assessment of tumor load in small laboratory animals. BLI is easy to perform, yielding a very reasonable throughput and an excellent sensitivity compared with other noninvasive imaging modalities. Cancer cell load can be determined from the moment that cancer cells are inoculated in the animals. When tumors are close to the surface or growing as a welldefined focal lesion, a good correlation between BLI signal and actual tumor mass is usually observed. Although this correlation is reduced in metastasis models in which multiple lesions grow relatively dispersed throughout the affected organ(s), changes in tumor mass relative to the initial BLI signal can still be accurately quantified when a uniform position of the animals toward the camera on the different imaging days is put into practice. These longitudinal data, with animals serving as their own control, provide information about the course of tumor development in asymptomatic animals and can lead to significant differences between experimental groups using relatively small groups of animals. These factors reduce both the number of animals as well as the discomfort they are exposed to. Although BLI does not provide information on morphology and/or growth patterns of individual tumors or interactions between cancer cells and host tissue, a combined approach of BLI and FLI, ultrasound, or MRI may overcome this. Moreover, postmortem macroscopical or microscopical analysis of the tissue that contains cancer cells guided by BLI images is another approach to link information on tumor load with structural and functional analysis of the tissues involved. Thus, BLI makes it possible to implement more advanced orthotopic tumor models into anticancer drug development screens with almost the same simplicity as when using ectopic 
subcutaneous models in combination with calipers.

\section{ACKNOWLEDGMENTS}

C.J.F.V.N. and O.V.T. contributed equally to the supervision of the study. This study was supported by grant ZonMW 3170.0056.

\section{REFERENCES}

1. Hanahan, D. and R.A. Weinberg. 2000. The hallmarks of cancer. Cell 100:57-70.

2.Lah, T.T., M.B. Durán Alonso, and C.J.F. Van Noorden. 2006. Antiprotease therapy in cancer: hot or not? Expert Opin. Biol. Ther. 6:257-279.

3. Garber, K. 2006. Realistic rodents? Debate grows over new mouse models of cancer. J. Natl. Cancer Inst. 98:1176-1178.

4. Killion, J.J., R. Radinsky, and I.J. Fidler. 1998. Orthotopic models are necessary to predict therapy of transplantable tumors in mice. Cancer Metastasis Rev. 17:279-284.

5. Hoffman, R.M. 1999. Orthotopic metastatic mouse models for anticancer drug discovery and evaluation: a bridge to the clinic. Invest. New Drugs 17:343-359.

6. Griffini, P., O. Fehres, L. Klieverik, I.M.C. Vogels, W. Tigchelaar, S.M. Smorenburg, and C.J.F. Van Noorden. 1998. Dietary $\Omega-3$ polyunsaturated fatty acid promote colon carcinoma metastasis in rat liver. Cancer Res. 58:3312-3319.

7.Lyons, S.K. 2005. Advances in imaging mouse tumour models in vivo. J. Pathol. 205:194-205.

8. Lewis, J.S., S. Achilefu, J.R. Garbow, R. Laforest, and M.J. Welch. 2002. Small animal imaging: current technology and perspectives for oncological imaging. Eur. J. Cancer 38:2173-2188

9.Zeamari, S., G. Rumping, B. Floot, S. Lyons, and F.A. Stewart. 2004. In vivo bioluminescence imaging of locally disseminated colon carcinoma in rats. Br. J. Cancer 90:1259-1264.

10. Mook, O.R.F., J. van Marle, H. VreelingSindelarova, R. Jonges, W.M. Frederiks, and C.J.F. Van Noorden. 2003. Visualization of early events in tumor formation of eGFPtransfected rat colon cancer cells in liver. Hepatology 38:295-304.

11.Di Nisio, M., A. Squizzato, C.P.W. Klerk, D.J. Richel, and H.R. Büller. 2004. Antithrombotic therapy and cancer. Curr. Opin. Hematol. 11:187-191.

12. Brown, E.B., R.B. Campbell, Y. Tsuzuki, L. Xu, P. Carmeliet, D. Fukumura, and R.K. Jain. 2001. In vivo measurement of gene expression, angiogenesis and physiological function in tumors using multiphoton laser scanning microscopy. Nat. Med. 7:864-868.

13. Wolf, K., I. Mazo, H. Leung, K. Engelke, U.H. Von Andrian, E.I. Deryugina, A.Y. Strongin, E.-B. Bröcker, and P. Friedl. 2003. Compensation mechanism in tumor cell migration: mesenchymal-amoeboid transition after blocking of pericellular proteolysis. J. Cell Biol. 160:267-277.

14. Ciancio, S.J., M. Coburn, and P.J. Hornsby. 2000. Cutaneous window for in vivo observations of organs and angiogenesis. J. Surg. Res. 92:228-232.

15. Yang, M., E. Baranov, J.W. Wang, P. Jiang, X. Wang, F.X. Sun, M. Bouvet, A.R. Moossa, et al. 2002. Direct external imaging of nascent cancer, tumor progression, angiogenesis, and metastasis on internal organs in the fluorescent orthotopic model. Proc. Natl. Acad. Sci. USA 99:3824-3829.

16. Koo, V., P.W. Hamilton, and K. Williamson. 2006. Non-invasive in vivo imaging in small animal research. Cell. Oncol. 28:127-139.

17. Hoffman, R.M. 2004. In vivo imaging with fluorescent proteins: the new cell biology. Acta Histochem. 106:77-87.

18. Hoffman, R. 2002. Green fluorescent protein imaging of tumour growth, metastasis, and angiogenesis in mouse models. Lancet Oncol. 3:546-556.

19. Tung, C.-H., Q. Zeng, K. Shah, D.-E. Kim, D. Schellingerhout, and R. Weissleder. 2004. In vivo imaging of $\beta$-galactosidase activity using far red fluorescent switch. Cancer Res. 64:1579-1583.

20. Yang, M., E. Baranov, P. Jiang, F.X. Sun, X.M. Li, L. Li, S. Hasegawa, M. Bouvet, et al. 2000. Whole-body optical imaging of green fluorescent protein-expressing tumors and metastases. Proc. Natl. Acad. Sci. USA 97:1206-1211.

21. Ponomarev, V., M. Doubrovin, I. Serganova, J. Vider, A. Shavrin, T. Beresten, A. Ivanova, L. Ageyeva, et al. 2004. A novel triple-modality reporter gene for whole-body fluorescent, bioluminescent, and nuclear noninvasive imaging. Eur. J. Nucl. Med. Mol. Imaging 31:740-751.

22. Jain, R.K., L.L. Munn, and D. Fukumura. 2002. Dissecting tumour pathophysiology using intravital microscopy. Nat. Rev. Cancer 2:266-276.

23. Wyckoff, J.B., J.G. Jones, J.S. Condeelis, and J.E. Segall. 2000. A critical step in metastasis: in vivo analysis of intravasation at the primary tumor. Cancer Res. 60:2504-2511.

24. Troy, T., D. Jekic-McMullen, L. Sambucetti, and B. Rice. 2004. Quantitative comparison of the sensitivity of detection of fluorescent and bioluminescent reporters in animal models. Mol. Imaging 3:9-23.

25. Ray, P., A. De, J.J. Min, R.Y. Tsien, and S.S. Gambhir. 2004. Imaging tri-fusion multimodality reporter gene expression in living subjects. Cancer Res. 64:1323-1330.

26. Weissleder, R. and V. Ntziachristos. 2003. Shedding light onto live molecular targets. Nat. Med. 9:123-128.

27. Edinger, M., T.J. Sweeney, A.A. Tucker, A.B. Olomu, R.S. Negrin, and C.H. Contag. 1999. Noninvasive assessment of tumor cell proliferation in animal models. Neoplasia 1:303-310.

28. Edinger, M., Y.A. Cao, Y.S. Hornig, D.E. Jenkins, M.R. Verneris, M.H. Bachmann, R.S. Negrin, and C.H. Contag. 2002. Advancing animal models of neoplasia through in vivo bioluminescence imaging Eur. J. Cancer 38:2128-2136.

29. Contag, C.H. and B.D. Ross. 2002. It's not just about anatomy: in vivo bioluminescence imaging as an eyepiece into biology. J. Magn. Reson. Imaging 16:378-387.

30. Szentirmai, O., C.H. Baker, N. Lin, S. Szucs, M. Takahashi, S. Kiryu, A.L. Kung, R.C. Mulligan, and B.S. Carter. 2006. Noninvasive bioluminescence imaging of luciferase expressing intracranial U87 xenografts: correlation with magnetic resonance imaging determined tumor volume and longitudinal use in assessing tumor growth and antiangiogenic treatment effect. Neurosurgery 58:365-372.

31. Kemper, E.M., W. Leenders, B. Küsters, S. Lyons, T. Buckle, A. Heerschap, W. Boogerd, J.H. Beijnen, and O. van Tellingen. 2006. Development of luciferase tagged brain tumour models in mice for chemotherapy intervention studies. Eur. J. Cancer 42:3294-3303.

32. Bhaumik, S. and S.S. Gamblin. 2002 Optical imaging of Renilla luciferase reporter gene expression in living mice. Proc. Natl. Acad. Sci. USA 99:377-382.

33. El Hilali, N., N. Rubio, M. MartinezVillacampa, and J. Blanco. 2002. Combined noninvasive imaging and luminometric quantification of luciferase-labeled human prostate tumors and metastases. Lab. Invest. 82:15631571.

34. Rosol, T.J., S.H. Tannehill-Gregg, B.E. LeRoy, S. Mandl, and C.H. Contag. 2003. Animal models of bone metastasis. Cancer 97:748-757.

35. Wetterwald, A., G. van der Pluijm, I. Que, B. Sijmons, J. Buijs, M. Karperien, C.W. Lowik, E. Gautschi, et al. 2002. Optical imaging of cancer metastasis to bone marrow: a mouse model of minimal residual disease. Am. J. Pathol. 160:1143-1153

36. Saur, D., B. Seidler, G. Schneider, H. Algül, R. Beck, R. Senekowitsch-Schmidtke, M. Schwaiger, and R.M. Schmid. 2005 CXCR4 expression increases liver and lung metastasis in a mouse model of pancreatic cancer. Gastroenterology 129:1237-1250.

37. Vooijs, M., J. Jonkers, S. Lyons, and A. Berns. 2002. Noninvasive imaging of spontaneous retinoblastoma pathway-dependent tumors in mice. Cancer Res. 62:1862-1867.

38. Douma, S., T. van Laar, J. Zevenhoven, R. Meuwissen, E. van Garderen, and D.S Peeper. 2004. Suppression of anoikis and induction of metastasis by the neurotrophic receptor TrkB. Nature 430:1034-1040.

39. Shah, K., Y. Tang, X. Breakefield, and R. Weissleder. 2003. Real-time imaging of TRAIL-induced apoptosis of glioma tumors in vivo. Oncogene 22:6865-6872.

40. Edinger, M., Y.-A. Cao, M.R. Verneris, M.H. Bachmann, C.H. Contag, and R.S. Negrin. 2003. Revealing lymphoma growth and the efficacy of immune cell therapies using in vivo bioluminescence imaging. Blood 101:640-648.

See Validity of bioluminescence... (Continued on page 30) 